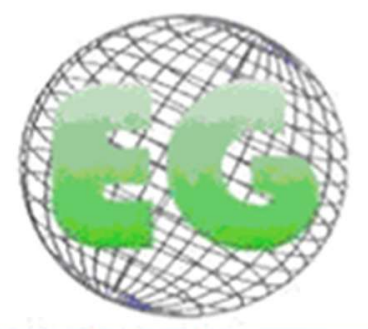

ISSN 1695-6141 $\mathbf{N}^{\circ} 63$

\title{
ORIGINALES
}

\section{La universidad como un entorno saludable: un estudio transversal}

The university as a healthy setting: a cross-sectional survey study

Ana López-Alonso ${ }^{1}$

Cristina Liébana-Presa ${ }^{2}$

Leticia Sánchez-Valdeón ${ }^{2}$

Mercedes López-Aguado ${ }^{3}$

Marta Quiñones-Pérez ${ }^{4}$

Elena Fernández-Martínez ${ }^{2}$

${ }^{1}$ Departamento de Enfermería y Fisioterapia. Grupo de investigación EDUSOC. Universidad de León, León, España.

2 Departamento de Enfermería y Fisioterapia. Grupo de investigación SALBIS. Universidad de León, Campus de Ponferrada, León, España. cliep@unileon.es

${ }^{3}$ Departamento de Psicología, Sociología y Filosofía. Grupo de investigación EDUSOC. Universidad de León, León, España

${ }^{4}$ Departamento de Enfermería y Fisioterapia. Universidad de León, León, España.

\section{https://doi.org/10.6018/eglobal.441601}

Recibido: $1 / 09 / 2020$

Aceptado: 10/01/2021

\section{RESUMEN:}

Introducción: Los estudiantes universitarios se encuentran en un momento crucial de sus vidas en lo que respecta a la adquisición de un estilo de vida saludable. Para esto es necesario que obtengan un equilibrio entre las demandas universitarias (compromiso académico) y el tiempo libre.

Objetivos: Describir las relaciones entre ocio, engagement y salud.

Método: Diseño de encuesta cuantitativa, no experimental. 1.142 estudiantes completaron los cuestionarios sobre ocio, salud y engagement. Se realizaron análisis descriptivos, de correlación lineal y ANOVA sobre los datos obtenidos.

Resultados: 960 estudiantes participaron en actividades de ocio activo con actividad física durante la semana (3-9 horas) y 1.021 los fines de semana (3-8 horas); 1.084 se dedicaba al ocio activo sin actividad física de lunes a viernes (4-14 horas) y 1.109 los fines de semana (7-16 horas); 1.049 se dedicaron al ocio pasivo todos los días (2-7 horas) y 997 los fines de semana (3-8 horas) de inactividad, mientras que 748 estuvieron inactivos durante la semana (1-5 horas) y 745 los fines de semana (2-4 horas). Las mujeres pasaron menos tiempo libre durante la semana, obtuvieron puntajes más altos de engagement y una peor salud percibida. Encontramos relación negativa entre la salud y el engagement y el ocio.

Conclusiones: Los estudiantes estaban comprometidos y gozaban de buena salud, lo que indica que un alto engagement y actividades de ocio están asociadas con una mejor salud percibida. Las universidades deben esforzarse por convertirse en entornos más saludables revisando sus planes de estudio, promoviendo el compromiso y ofreciendo un programa de ocio atractivo. 
Palabras clave: Salud; Estudiantes universitarios; Engagement; Ocio; Salud del estudiante; Estilo de vida.

\begin{abstract}
:
Introduction: University students are at a crucial time in their lives as regards acquiring a healthy lifestyle. For this it is necessary that they obtain a balance between the times used to the university demands (academic engagement) and the leisure time, as recovery time.

Objectives: To describe the relationships between leisure, engagement and health.

Method: We employed a quantitative, non-experimental survey design. 1.142 students completed the questionnaires on leisure, health and engagement. Descriptive, linear correlation and ANOVA analyses were performed on the data obtained.

Results: 960 students engaged in active leisure with physical activity during the week (3-9 hours) and 1.021 at the weekend (3-8 hours); 1.084 engaged in active leisure without physical activity from Monday to Friday (4-14 hours) and 1.109 at the weekend (7-16 hours); 1.049 engaged in passive leisure every day (2-7 hours) and 997 at the weekend (3-8 hours) idleness, while 748 were idle during the week (1-5 hours) and 745 at the weekend (2-4 hours). Women spent less time of leisure during the week, scored higher of engagement and had poorer perceived health. We found a negative relationship between health and engagement and leisure.

Conclusions: Students were engaged and in good health, indicating that high engagement and leisure activities are associated with better perceived health. Universities should strive to become healthier settings by revising their curricula, promoting engagement and offering an attractive leisure programme.
\end{abstract}

Key words: Health; University students; Engagement; Leisure activities; Student health; Life style

\title{
INTRODUCCIÓN
}

La Organización Mundial de la Salud (OMS), en su informe de Salud para todos en el siglo XXI de la región europea ${ }^{(1)}$, establece que la salud debe ser promovida en los escenarios saludables y define estos como lugares donde la gente vive, trabaja o se divierte.

Además de su función académica, las universidades también pueden influir en la salud; la vida universitaria conlleva actividades diarias que incluyen factores ambientales, organizativos y personales que repercuten en el bienestar. Por lo tanto, es importante que las universidades promuevan la salud entre las personas que trabajan y estudian allí, de acuerdo con las estrategias diseñadas por la OMS. Como entorno de salud, las universidades se consideran instituciones de promoción de la salud, definidas como lugares que refuerzan constantemente su capacidad como lugares saludables para vivir, aprender y trabajar ${ }^{(2)}$.

Paralelamente al proyecto "Ciudades Saludables" de la OMS, como manifestación de "Salud para todos" en el ámbito de la ciudad, se crea la Red Española de Ciudades Saludables ${ }^{(3)}$ que incluye el proyecto "Universidades Saludables" a partir del cual se instaura la "Red Española de Universidades Saludables" (REUS) $)^{(4)}$. El principio fundamental es que la salud está fuertemente influenciada por el entorno en el que los estudiantes universitarios viven, trabajan, estudian, comen, se mueven y se relajan. En línea con la OMS y con la idea de promoción de la salud, las universidades que se han unido al REUS y han lanzado un plan estratégico que refleja la importancia de implementar acciones dirigidas a fortalecer la capacidad de las personas para modificar los determinantes sociales, ambientales y económicos de la salud, según lo establecido en la Carta de Ottawa de $1987^{(5)}$. Los objetivos de REUS destacan el papel de las universidades como promotoras de la salud y el bienestar en la comunidad universitaria y de la sociedad en su conjunto. Uno de sus enfoques 
estratégicos es "ofrecer servicios y actividades destinados a promover la salud entre la comunidad universitaria"(4).

Las universidades que se esfuerzan por ofrecer una educación de calidad deben tener en cuenta las variables relacionadas con el proceso de enseñanza-aprendizaje ${ }^{(6)}$, una de las más importantes es la salud.

Durante años se ha surgido al preocupación por conocer si los planes de estudios están sobredimensionados de tal manera que los estudiantes soportan una carga horaria incluso superior a una jornada de trabajo(7). A priori, esto implica que los estudiantes universitarios carecen de tiempo libre para realizar actividades de ocio. Un sistema educativo debe incluir el tiempo libre, ya que para mantener la salud y desempeñarse bien, es necesario alternar los períodos de actividad con períodos de descanso $^{(8)}$ y especialmente el descanso activo ${ }^{(9,10)}$. Esto es particularmente cierto en el caso de los estudiantes universitarios, que no solo tienen que aprender a administrar su tiempo de estudio sino también su tiempo libre ${ }^{(11)}$. En general, debido a que son jóvenes, los estudiantes universitarios consideran que su tiempo libre es uno de los aspectos más importantes de sus vidas, ya que les permite relajarse, recuperarse ${ }^{(12)}$ y también construir una red de relaciones sociales cercanas ${ }^{(9)}$. Estos, a su vez, de acuerdo con estudios de análisis de redes sociales, influyen como grupo de apoyo en el compromiso con sus estudios universitarios ${ }^{(13)}$, sabiendo que sus interacciones personales, intervienen en diferentes situaciones de aprendizaje ${ }^{(14)}$. Este impulso interno para invertir ese esfuerzo se conoce como engagement. El concepto de participación de los estudiantes es similar al de vinculación psicológica en el trabajo(15) y, por extensión, en los estudios ${ }^{(16)}$. Por otro lado, la investigación indica que practicar alguna actividad física (ocio activo) influye positivamente tanto en el rendimiento académico como en la autoeficacia de los estudiantes, promoviendo un estilo de vida saludable y un buen rendimiento académico ${ }^{(17)}$.

Actualmente, las universidades están experimentando múltiples cambios que requieren que los estudiantes se adapten y respondan constantemente. Por lo tanto, es interesante explorar los factores que influyen en las actitudes positivas hacia el cambio que motivan a los estudiantes a enfrentar y superar desafíos continuos, perseverar en su ambición de completar su carrera elegida, buscar un equilibrio entre el estudio y el tiempo libre y mantener una buena salud mientras están en universidad(18). Por todas estas razones, la implementación de medidas preventivas organizacionales en las universidades puede ser importante para garantizar un desempeño académico saludable y favorable para los estudiantes.

El objetivo del presente estudio fue describir y analizar las relaciones y asociaciones que existen entre las actividades de ocio, el engagement y la salud de los estudiantes universitarios.

\section{MATERIAL Y MÉTODO}

\section{Participantes}

Se realiza un diseño cuantitativo no experimental de encuesta. La población de estudio fue de 1.749 estudiantes pertenecientes a seis disciplinas diferentes de una Universidad Pública de España. Los participantes comprendieron 1.142 estudiantes, de los cuales el $37 \%$ eran hombres y el $63 \%$ mujeres. La Tabla 1 muestra la 
distribución por sexo para cada una de las disciplinas encuestadas. La edad de los participantes osciló entre un mínimo de 18 años y un máximo de 52 años, con una media de 21.7 años.

Tabla 1. Descriptivos de muestra

\begin{tabular}{llll}
\hline Titulación & Género & \multicolumn{2}{l}{ Total n (\%) } \\
\cline { 2 - 3 } & Masculino & Femenino & \\
\hline Enfermería (Campus 1) & 31 & 239 & $270(24 \%)$ \\
\hline Enfermería (Campus 2) & 22 & 96 & $118(10 \%)$ \\
\hline Educación Infantil & 4 & 124 & $128(11 \%)$ \\
\hline Psicopedagogía & 7 & 33 & $40(3 \%)$ \\
\hline Ciencias de la Actividad Física y Deporte & 204 & 100 & $304(27 \%)$ \\
\hline Ingeniería & 119 & 42 & $161(14 \%)$ \\
\hline Fisioterapia & 35 & 86 & $121(11 \%)$ \\
\hline Total & $422(37 \%)$ & $720(63 \%)$ & $1142(100 \%)$ \\
\hline
\end{tabular}

\section{Instrumentos de recogida de datos}

El Cuestionario de Tiempo Libre y Ocio (ETLO), fue adaptado de los cuestionarios desarrollados por Ponce de León ${ }^{(19)}$ y Sánchez-Herrero et al.(20). Este cuestionario ha sido evaluado favorablemente en una prueba piloto desarrollada por un comité de expertos $^{(21)}$. El cuestionario ETLO presenta una lista de actividades específicas asociadas con las categorías ocio activo con actividad física ( $A L+P A)$, ocio activo sin actividad física (AL-PA), ocio pasivo (PL) e inactividad (ID). El ocio activo se refiere a las actividades en las que la persona es la protagonista con una participación sustancial del sistema cardiorrespiratorio y la coordinación neuromuscular. Ocio activo sin actividad física, incluye actividades artísticas, literatura, juegos de mesa, entre otros. El ocio pasivo se entiende como aquello en que la persona es un mero receptor o espectador de la acción de los demás, y finalmente, la ociosidad o la inactividad, no hace nada. Se pide a los encuestados que estimen el tiempo (en horas y minutos) que dedican a 19 actividades de ocio durante la semana (MF) y los fines de semana (WE).

El Cuestionario de salud general $(\mathrm{GHQ}-12)^{(22)}$ es una herramienta de detección psicométrica desarrollada para identificar la salud percibida $(\alpha=0.85)$. Los encuestados calificaron cada uno de los 12 ítems utilizando una escala de tipo Likert de 4 puntos, donde una puntuación más alta indica una salud percibida más pobre

La Escala de Engagement para estudiantes (UWES-Estudiante)(23) validada en la población española ${ }^{(16)}$ consta de 17 ítems que miden las tres dimensiones del constructo: vigor $(\alpha=0.85)$, dedicación $(\alpha=0.83)$ y absorción $(\alpha=0.84)$. El vigor se refiere a los altos niveles de energía con las tareas realizadas. La dimensión de dedicación responde a altos niveles de significado en las tareas realizadas. Finalmente, la absorción implica altos niveles de concentración y felicidad mientras se realiza una actividad. Los encuestados calificaron cada ítem utilizando una escala tipo Likert de 7 puntos. Las puntuaciones altas indican fuertes niveles de engagement.

\section{Procedimiento}

Después de obtener el consentimiento de los estudiantes para participar voluntariamente, los datos se recopilaron utilizando la herramienta en línea LimeSurvey. Los datos fueron recolectados en presencia del investigador principal. Se 
explicó el significado de cada variable, homogeneizando así su comprensión entre los participantes. Una vez que se completaron los cuestionarios, todos los estudiantes que lo solicitaron fueron informados de los objetivos y el procedimiento del estudio.

\section{Análisis de datos}

Los análisis estadísticos se realizaron con SPSS 26.0. Se realizó análisis de correlación descriptivos y lineales utilizando el coeficiente de correlación de Pearson. Para determinar la existencia de diferencias significativas entre los grupos, se realizó un análisis de varianza ANOVA. Además, la fiabilidad de los instrumentos se calcula utilizando el coeficiente alfa de Cronbach, que mide la consistencia interna.

\section{Consideraciones éticas}

Las encuestas fueron acompañadas por una hoja de información y consentimiento por escrito. En todo momento se consideró el anonimato de los sujetos que formaron parte del estudio y también se aseguró de que la participación fuera voluntaria. Durante el estudio, se siguieron las pautas nacionales e internacionales (Código de Ética y Declaración de Helsinki), y seguimos las regulaciones legales sobre confidencialidad de datos. El estudio fue aprobado por el comité de ética de la Universidad pública, lo que garantiza el cumplimiento de las cuestiones éticas y legales.

\section{RESULTADOS}

La Tabla 2 muestra las estadísticas descriptivas de los estudiantes que llevaron a cabo las actividades enumeradas (ocio activo con actividad física, ocio activo sin actividad física, ocio pasivo e inactividad) y el tiempo que dedicaron a cada una (de lunes a viernes y fin de semana).

Tabla 2. Valores descriptivos para actividades de ocio.

\begin{tabular}{|c|c|c|c|c|c|}
\hline & & $\begin{array}{l}\text { Estudiantes } \\
\mathbf{N}(\%)\end{array}$ & Tiempo mínimo & Tiempo máximo & $\mathrm{M} \pm \mathrm{DT}$ \\
\hline \multirow{4}{*}{$\frac{\mathbf{L}}{\Sigma}$} & $A L+P A$ & $960(84.1 \%)$ & 0.17 & 35 & $6.7 \pm 5$ \\
\hline & AL-PA & $1.084(4.9 \%)$ & 0.25 & 148.5 & $10.4 \pm 9.4$ \\
\hline & $\mathrm{PL}$ & $1.049(91.9 \%)$ & 0.02 & 32 & $5.7 \pm 4.5$ \\
\hline & ID & $748(65.5 \%)$ & 0.02 & 40 & $3.4 \pm 3.6$ \\
\hline \multirow{4}{*}{ W } & $A L+P A$ & $1.021(89.4 \%)$ & 0.33 & 35 & $6 \pm 4.5$ \\
\hline & AL-PA & $1.109(91.7 \%)$ & 0.07 & 69 & $12.2 \pm 8.1$ \\
\hline & $\mathrm{PL}$ & $997(87.3 \%)$ & 0.05 & 35 & $5.1 \pm 3.5$ \\
\hline & ID & $745(65 \%)$ & 0.02 & 304 & $3.5 \pm 2.9$ \\
\hline
\end{tabular}

Note: $\mathrm{M} \pm \mathrm{DT}$ : Media \pm Desviación típica. $\mathrm{AL}+\mathrm{PA}$ : ocio activo con actividad física. AL-PA: ocio activo sin actividad física. PL: ocio pasivo. ID: inactividad. MF: Lunes a Viernes. WE: Fin de semana.

El análisis de la Tabla 2 muestra que el mayor porcentaje de estudiantes dedicaron su tiempo libre al ocio activo sin actividad física, tanto durante la semana como durante el fin de semana. Alrededor del $50 \%$ de los encuestados dedicaron entre cuatro y catorce horas a este tipo de actividad durante la semana y entre siete y dieciséis horas los fines de semana. De lunes a viernes, estas actividades fueron seguidas en segundo lugar por actividades de ocio pasivo, que ocuparon el tercer lugar el fin de 
semana, con un $50 \%$ de gasto entre dos y siete horas en estas actividades durante la semana y entre tres y ocho horas los fines de semana. La categoría de ocio activo con actividad física ocupó el tercer lugar durante la semana y el segundo lugar el fin de semana, con un $50 \%$ de gasto entre tres y nueve horas durante la semana y entre tres y ocho horas los fines de semana. La categoría de ociosidad ocupó el último lugar de lunes a viernes y durante el fin de semana, con un $50 \%$ de los estudiantes pasando entre una y cinco horas durante la semana y entre dos y cuatro horas los fines de semana.

En general, el tiempo libre aumentó durante el fin de semana, al igual que todas las formas de ocupación o no ese tiempo. Sin embargo, este aumento no fue proporcional en todas las categorías; en cambio, considerando el rango del $25 \%$ más bajo, $50 \%$ y el $25 \%$ más alto, se puede ver que el mayor aumento durante el fin de semana ocurrió con el ocio activo sin actividad física. El tiempo dedicado al resto de las categorías de ocio se mantuvo más o menos estable.

Como se muestra en la Figura 1, de lunes a viernes, las mujeres pasaron menos tiempo que los hombres en todas las categorías de actividades de ocio, y estas diferencias fueron estadísticamente significativas $(p<0.05)$. Por el contrario, durante el fin de semana, no se detectaron diferencias estadísticamente significativas entre hombres y mujeres en ninguna de las categorías.

Figura 1. Distribución de los tiempos y relación con el género, según las actividades de ocio categorizadas.

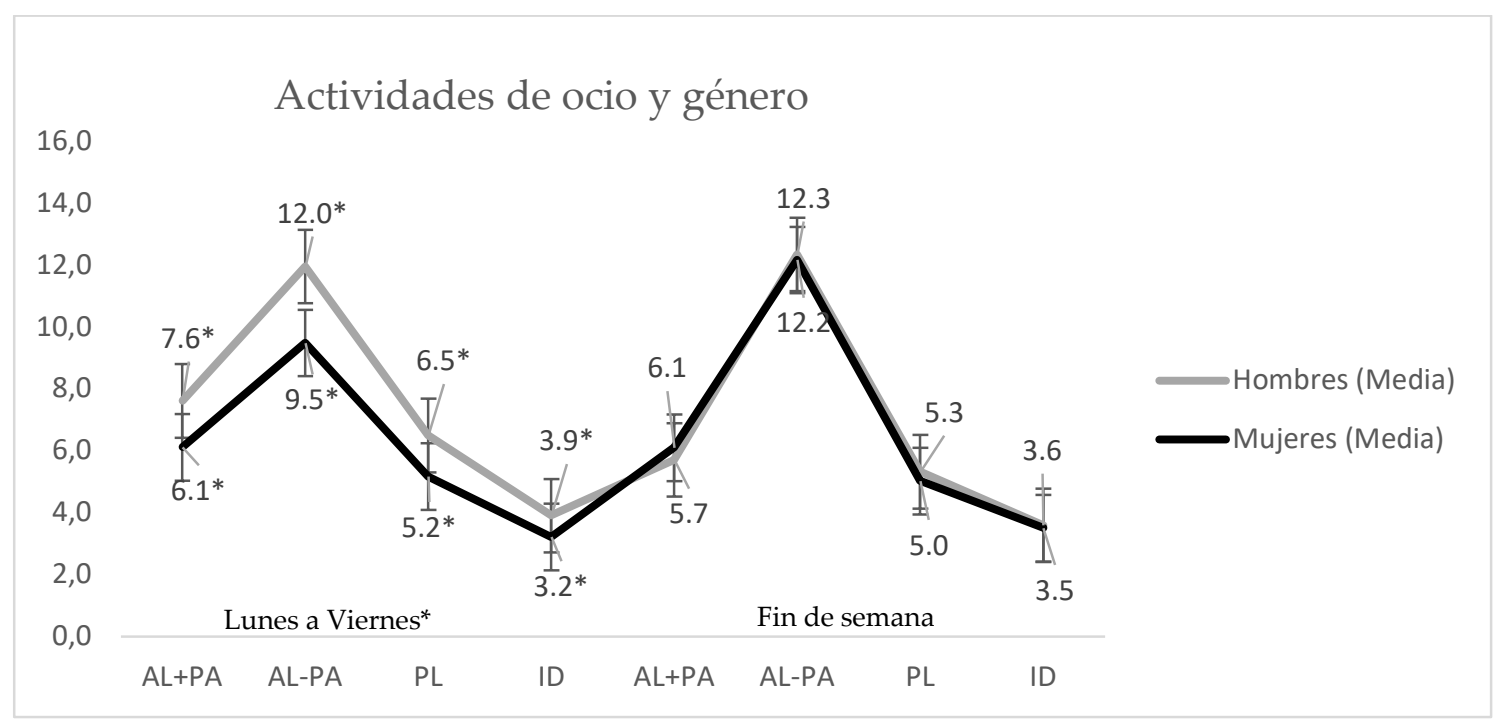

Nota: *p <0.05(ANOVA). AL + PA: ocio activo con actividad física. AL-PA: ocio activo sin actividad física. PL: ocio pasivo. ID: inactividad.

Como muestra la prueba ANOVA, las mujeres obtuvieron puntuaciones medias en el valor total y en todas las dimensiones del engagement, significativamente más altas ( $p$ $<0.05$ ) que los hombres (Tabla 3). 
Tabla 3. ANOVA de engagement en relación con mujeres y hombres con respecto a la edad

\begin{tabular}{llll}
\hline Engagement & Género & Edad & p-valor \\
\hline Vigor & Hombres & $3.46 \pm 1.31$ & 0.001 \\
\cline { 2 - 4 } & Mujeres & $3.85 \pm 1.36$ & \\
\hline Dedicación & Hombres & $4.66 \pm 1.19$ & 0.016 \\
\cline { 2 - 4 } & Mujeres & $4.99 \pm 1.14$ & \\
\hline Absorción & Hombres & $3.43 \pm 1.24$ & 0.008 \\
\cline { 2 - 4 } & Mujeres & $3.89 \pm 1.30$ & \\
\hline Total & Hombres & $3.85 \pm 1.10$ & 0.001 \\
\cline { 2 - 4 } & Mujeres & $4.24 \pm 1.12$ & \\
\hline
\end{tabular}

Con respecto al GHQ-12, el puntaje promedio fue de 12.74 con una desviación estándar de 5.58. Estos valores reflejan muy buena salud percibida. Las mujeres obtuvieron puntajes significativamente más altos que los hombres (13.48 vs 11.47; $p$ $<0.05$ ), lo que indica que tenían un bienestar percibido más pobre.

La relación entre las actividades de salud y ocio, los resultados de las correlaciones mostraron una tendencia negativa para todas las actividades. Por lo tanto, cuanto más tiempo se dedica a las diferentes actividades, menor es el puntaje GHQ-12 y, por lo tanto, mejor se percibe la salud. Estas relaciones fueron estadísticamente significativas para las variables ocio activo con actividad física ( $r=-0.112)$, ocio activo sin actividad física $(r=-0.092)$ y ocio pasivo $(r=-0.080)$, de lunes a viernes, y ocio activo sin actividad física $(r=-0.111)$ los fines de semana $(p<0.01)$.

Las diferencias en las medias (ANOVA) también fueron estadísticamente significativas $(p<0.01)$ en un análisis de grupos con mayor y menor percepción de salud (menor $\leq 8$, mayor $\geq 16$ ) para las variables ocio activo con actividad física durante la semana y ocio activo sin actividad física los fines de semana (Tabla 4). Los valores de GHQ-12 se clasifican en tres grupos. El primero y el más bajo se refieren a los valores del percentil 25 más bajo (peor percepción de salud). En segundo lugar, los valores más altos se refieren al percentil 25 superior (mejor salud percibida) y el tercer grupo se refiere a los puntajes intermedios (salud percibida promedio). Por lo tanto, los estudiantes que se dedicaron al ocio activo con actividad física durante la semana presentaron una mejor salud percibida. Lo mismo sucedió con los estudiantes que se dedicaron al ocio activo sin actividad física durante el fin de semana. Independientemente de la categoría, el ocio se asoció con una mejor salud percibida.

Tabla 4. ANOVA de salud general en relación con el ocio activo con y sin actividad física

\begin{tabular}{|c|c|c|c|}
\hline \multicolumn{2}{|c|}{ Salud percibida (GHQ-12) } & \multirow{2}{*}{$\begin{array}{l}\text { MF ocio activo } \\
\text { actividad física } \\
5.79 \pm 4.16\end{array}$} & \multirow{2}{*}{$\begin{array}{l}\text { WE ocio activo } \\
\text { actividad física } \\
11.25 \pm 7.50\end{array}$} \\
\hline Peor percepción & $\mathrm{M} \pm \mathrm{SD}$ & & \\
\hline salud( $(\leq 8)$ & (n) & 228 & 286 \\
\hline Salud percibida & $\mathrm{M} \pm \mathrm{SD}$ & $6.80 \pm 5.29$ & $12.08 \pm 8.33$ \\
\hline promedio (8-16) & $(n)$ & 471 & 544 \\
\hline Mejor salud percibida & $\mathrm{M} \pm \mathrm{SD}$ & $7.43 \pm 5.01$ & $13.52 \pm 8.31$ \\
\hline$(\geq 16)$ & (n) & 261 & 279 \\
\hline p-value & & 0.001 & 0.004 \\
\hline
\end{tabular}

Nota: M: Media. DT: Desviación típica. MF: Lunes a Viernes. WE: Fin de semana. 
La salud se relacionó negativamente con el engagement $(r=-0.102, p=0.01)$, la dedicación $(r=-0.139, p=0.01)$ y el vigor $(r=-0.112, p=0.01)$. Esta tendencia negativa en la correlación indica que cuanto mayor sea el puntaje de engagement, dedicación y vigor, menor será la puntuación para el GHQ-12; por lo tanto, los estudiantes que obtuvieron valores más altos en engagement presentaron mejor salud percibida. Los resultados del ANOVA (Tabla 5) con mayor, promedio y menor percepción de salud (menor $\leq 8$, mayor $\geq 16$ ) reflejaron esta tendencia, con diferencias significativas entre las medias $(p<0.01)$. Por lo tanto, los estudiantes con mayor vigor y dedicación presentaron una mejor salud percibida.

Tabla 5. ANOVA de salud general en relación al engagement (vigor y dedicación)

\begin{tabular}{|c|c|c|c|c|}
\hline GHQ-12 & & Vigor & Dedicación & Engagement \\
\hline \multirow{2}{*}{$\begin{array}{l}\text { Peor percepción de salud ( } \leq \\
\text { 8) }\end{array}$} & $\mathrm{M} \pm \mathrm{SD}$ & $3.52 \pm 1.35$ & $4.62 \pm 1.31$ & $3.94 \pm 1.18$ \\
\hline & (n) & 298 & 298 & 298 \\
\hline \multirow{2}{*}{$\begin{array}{l}\text { Salud percibida promedio (8- } \\
\text { 16) }\end{array}$} & $\mathrm{M} \pm \mathrm{SD}$ & $3.66 \pm 1.33$ & $4.92 \pm 1.10$ & $4.09 \pm 1.10$ \\
\hline & (n) & 558 & 558 & 558 \\
\hline \multirow{2}{*}{$\begin{array}{l}\text { Mejor salud percibida } \\
(\geq 16)\end{array}$} & $\mathrm{M} \pm \mathrm{SD}$ & $3.97 \pm 1.37$ & $5.02 \pm 1.10$ & $4.28 \pm 1.12$ \\
\hline & (n) & 286 & 286 & 286 \\
\hline p-valor & & 0.000 & 0.000 & 0.002 \\
\hline
\end{tabular}

Nota: M: Media. DT: Desviación típica

\section{DISCUSIÓN}

Aunque la mayoría de los estudios se han realizado en poblaciones con diferentes edades y no hay consenso sobre cómo clasificar las diferentes actividades de ocio en una población joven, se ha demostrado que las personas más jóvenes tienden a participar en las mismas actividades ${ }^{(21)}$ y que es la práctica de actividades de ocio con ejercicio físico las que están relacionadas con mejores resultados académicos ${ }^{(17)}$, con una mejor recuperación ${ }^{(12)}$ y una mayor percepción de la salud ${ }^{(24)}$. Se podrían realizar análisis adicionales sobre las diferencias obtenidas por sexo para adaptar las intervenciones a las necesidades específicas de hombres y mujeres, ya que de acuerdo con diferentes investigaciones ${ }^{(20)}$, hombres y mujeres presentan diferentes preferencias de actividades de ocio. Nuestros resultados indican que, si bien los hombres y las mujeres presentan diferentes valores para el ocio activo con o sin actividad física, ocio pasivo y ociosidad, ambos sexos participan claramente en el mismo tipo de actividades, pero en diferente medida, según las características de la población joven estudiada(25). Estos resultados son coincidentes con los reportados en estudios previos ${ }^{(26)}$, donde más de la mitad de la población joven analizada realizaba actividad física regularmente además de escuchar música y mirar la televisión como otras actividades diarias de ocio que los jóvenes realizaban con mayor frecuencia.

Nuestros datos sugieren que la presente muestra de estudio consistió en estudiantes comprometidos (engaged), motivados e implicados con sus estudios, además, igual que en otras investigaciones, presentan capacidad creativa para hacer frente con éxito al cambio(18). Los valores obtenidos son similares a los informados en otros estudios realizados en entornos universitarios ${ }^{(13,27)}$. En relación con la edad, el género y el engagement, varios estudios han encontrado diferencias significativas, por lo que los estudiantes mayores y las mujeres obtienen valores más altos para el engagement ${ }^{(27)}$; Los resultados del presente estudio confirman estas diferencias. 
De acuerdo con otros estudios, encontramos que los estudiantes en general presentaron buena salud percibida(27,28) y, en particular, el grupo de mujeres, informaron peor salud percibida ${ }^{(27)}$.

Los estudiantes que obtuvieron valores altos en el total de engagement, vigor y dedicación obtuvieron puntuaciones bajas en la escala de salud de Goldberg, lo que indica una mejor salud percibida. Estos resultados son coincidentes con los conseguidos por otros autores ${ }^{(29)}$, quienes han argumentado que el compromiso desencadena actitudes positivas y esto a su vez mejora la salud y el bienestar de las personas.

En los últimos años, el tiempo libre y el ocio, y especialmente el ocio activo con o sin actividad física, se han relacionado directamente con la salud, debido al papel protector que desempeñan contra el daño a la salud. Se ha demostrado que la actividad física es muy efectiva para promover la salud( ${ }^{(30)}$ también, mejora el rendimiento académico, la autoeficacia y promueve estilos de vida saludables ${ }^{(17)}$, incluso es un buen predictor de estas variables ${ }^{(25)}$. El presente estudio confirma la existencia de una relación entre el ocio y la salud percibida. El ocio activo con actividad física durante la semana fue la categoría que mejoró la salud percibida, en esa línea, los resultados de este estudio coinciden con los obtenidos de otra investigación, que indican los beneficios de utilizar el tiempo libre para dedicarse al ocio activo con actividad física(17). Nuestros hallazgos van más allá, revelando que es el ocio en general lo que influye en una mejor salud percibida, incluso cuando se trata de un ocio pasivo, siempre que no sea el principal o el único tipo, debido a su efecto restaurador y de recuperación ${ }^{(12)}$.

Esta investigación presenta limitaciones, es por ello que los resultados deben interpretarse con cautela. La población de estudio no es representativa de los estudiantes universitarios. Solo se obtuvieron datos sobre estudiantes de cursos de seis grados de la misma universidad, aunque de diferentes disciplinas (humanistas y técnicas) y de diferentes duraciones. En futuras investigaciones, sería interesante incluir una gama más amplia de cursos de grado para obtener resultados más representativos. Además, la naturaleza transversal de esta investigación, hizo imposible hacer inferencias causales sobre las relaciones detectadas. La composición de los estudiantes en la muestra de estudio presentó un claro sesgo hacia las mujeres.

\section{CONCLUSIONES}

Este estudio subraya la importancia de la universidad como un entorno saludable. Las intervenciones de estilo de vida se encuentran entre las acciones más efectivas en términos de prevención de enfermedades y promoción de la salud, y los resultados indican que promover el ocio activo con actividad física mejoraría la salud percibida en estos universitarios.

Nuestros hallazgos resaltan la importancia del ocio como un factor en la compensación de recuperación que se asocia con una mejor salud percibida. Los estudiantes que presentan una mejor recuperación están más comprometidos (engaged) con sus estudios, lo que cierra el círculo entre estas variables, y esto a su vez contribuye a un mayor éxito en la universidad y en el ámbito personal y profesional. 
En consecuencia, las universidades deberían reflexionar sobre los planes de estudio que exigen horas excesivas para no sobrecargar a los estudiantes. Programar, promover e incentivar actividades de ocio atractivas y saludables, principalmente aquellas que incluyen ejercicio físico, sería efectivo para garantizar una recuperación completa y estilos de vida saludables.

\section{REFERENCIAS}

1. World Health Organization Regional Office for Europe. Health 2020: A European policy framework and strategy for the 21st century [Internet]. Copenhagen; 2013 [cited 2020 Apr 10]. 190 p. Available from: http://www.euro.who.int/_data/assets/pdf_file/0011/199532/Health2020-Long.pdf 2. Martínez-Riera JR, Gallardo Pino $\bar{C}$, Aguiló Pons A, Granados Mendoza MC, López-Gómez J, Arroyo Acevedo HV. La universidad como comunidad: universidades promotoras de salud. Informe SESPAS 2018. Gac Sanit. 2018;32(S1):86-91.

3. Llorca E, Amor MT, Merino B, Márquez FJ, Gómez F, Ramírez R. Healthy cities: A reference strategy in local public health policies. Gac Sanit. 2010;24(6):4356.

4. Red Española de Universidades Saludables (REUS). La Universidad como entorno promotor de salud. [Internet]. 2011 [cited 2019 May 12]. Available from: https://www.unisaludables.es/es/

5. World Health Organization (WHO). WHO Traditional Medicine Strategy 20142023. World Heal Organ [Internet]. 2013, 1-76. Available from: http://apps.who.int/iris/bitstream/10665/92455/1/9789241506090_eng.pdf?ua=1

6. De-la-Fuente J, Zapata L, Martínez-Vicente JM, Sander $\overline{\mathrm{P}}$, Cardelle-Elawar M. The role of personal self-regulation and regulatory teaching to predict motivationalaffective variables, achievement and satisfaction: A structural model. Front Psychol. 2015;6(339).

7. Tembo C, Burns S, Kalembo F. The association between levels of alcohol consumption and mental health problems and academic performance among young university students. PLoS One. 2017;12(6):e0178142.

8. BurgessA, Senior, C, Moores E. A 10-year case study on the changing determinants of university student satisfaction in the UK. PLoS One. 2018;13: e0192976.

9. Lazcano Quintana I, Madariaga Ortuzar A. The leisure experience of young people with disabilities. Pedagog Soc Rev Interuniv. 2018;31: 109-121.

10. Sandoval N. Diagnosis about the use of leisure and free time among the students of the university. Pedagog Soc Rev Interuniv. 2017; 30: 163-180.

11. García-Cué JL, Santizo JA. Análisis de la relación entre la gestión del tiempo libre, el ocio y los estilos de aprendizaje. Rev Estilos Aprendiz. 2010;3(5):2-25.

12. Sonnentag S, Venz L, Casper A. Advances in recovery research: What have we learned? What should be done next? J Occup Health Psychol. 2017; 22(3): 365-80.

13. Fernández-Martínez E, Andina-Díaz E, Fernández-Peña R, García-López R, Fulgueiras-Carril I, Liébana-Presa C. Social Networks, Engagement and Resilience in University Students. Int. J. Environ. Res. Public Health. 2017; 14(12): 1488. doi: 10.3390/ijerph14121488.

14. De la Fuente J, La Hortiga-Ramos F, Laspra-Solís C, Maestro-Martín C, Alustiza I, Aubá E, et al. A structural equation model of achievement emotions, coping strategies and engagement-burnout in undergraduate students: A possible underlying 
mechanism in facets of perfectionism. Int J Environ Res Public Health. 2020 ;17(6):2106. doi: 10.3390/ijerph17062106.

15. Keyko K, Cummings GG, Yonge O, Wong CA. Work engagement in professional nursing practice: A systematic review. Int J Nurs Stud. 2016; 61: 142164.

16. Schaufeli W, Bakker A. UWES-Utrecht Work Engagement Scale. Preliminary Manual. Escala Utrecht de Engagement en el trabajo. Occupational Health Psychology Unit Utrecht University, 2009. Available from: http://scholar.google.com/scholar?hl=en\&btnG=Search\&q=intitle:Utrecht+work+engag ement+scale\#0

17. Keating XD, Castelli D, Ayers SF. Association of weekly strength exercise frequency and academic performance among students at a large university in the United States. J Strength Cond Res. 2013: 27(7); 1988-93.

18. Duncan JM, Withers MC, Lucier-Greer M, Ferraro AJ, Reed-Fitzke K. Research note: social leisure engagement, peer support, and depressive symptomology among emerging adults. Leisure Studies. Routledge. 2018; 37(3): 343-51. doi:10.1080/02614367.2017.1411968.

19. Ponce de León A. Tiempo libre y rendimiento académico. [Internet]. La Rioja: Universidad de La Rioja; 1998. 12. Available from: https://dialnet.unirioja.es/servlet/libro?codigo $=12786$

20. Sánchez-Herrero Arbide S. La importancia de la perspectiva de género en la psicología del ocio. An Psicol. 2008, 24, 64-76.

21. López-Alonso Al, López-Aguado M, González-Millán I, Fernández-Martínez E. Leisure and approaches to learning in nursing students. Rev Investig Educ. 2012;30(1):53-70.

22. Sánchez-López $M$ del $P$, Dresch $V$. The 12-item general health questionnaire (GHQ-12): Reliability, external validity and factor structure in the Spanish population. Psicothema. 2008; 20: 839-843.

23. Schaufeli WB, Salanova M, González-Romá V, Bakker AB. The measurement of engagement and burnout: a two sample confirmatory factor analytic approach. J Happiness Stud. 2002;3(1):71-92.

24. López-Alonso Al, López-Aguado M, Fernández-Martínez ME, Liébana-Presa C, Gutiérrez-Provecho ML. Los enfoques de aprendizaje, el "engagement", el ocio y el rendimiento anterior. Propuesta de un modelo. Bordón Rev Pedagog. 2016;68(4):6788

25. Lanuza Brosed R, Ponce de León Eliozondo A, Sanz Arazuri E, Valdemoros San Emeterio MÁ. La clase de educación física escolar como generadora de un ocio físico-deportivo. Retos Nuevas tendencias en Educ Física, Deport y Recreación. 2012; 22:13-5.

26. Clemente FM, Nikolaidis PT, Martins FML, Mendes RS. Physical activity patterns in university students: Do they follow the public health guidelines? PLoS One. 2016;11(3): e0152516.

27. Fernández-Martínez E, López-Alonso Al, Marqués-Sánchez $P$, MartínezFernández MC, Sánchez-Valdeón L, Liébana-Presa C. Emotional intelligence, sense of coherence, engagement and coping: A cross-sectional study of university students' health. Sustain. 2019;11(24): 6953. doi:10.3390/su11246953.

28. De-Mateo-Silleras B, Camina-Martín MA, Cartujo-Redondo A, Carreño-Enciso L, de-la-Cruz-Marcos S, Redondo-del-Río P. Health Perception According to the Lifestyle of University Students. J Community Health. 2019;44(1):74-80. 
29. Carrasco AM, de la Corte de la Corte CM, León Rubio JM. Engagement: un recurso para optimizar la salud psicosocial en las organizaciones y prevenir el burnout y estrés laboral. Rev Digit Prevención. 2010;1(1):1-22.

30. Calestine J, Bopp M, Bopp CM, Papalia Z. College Student Work Habits are Related to Physical Activity and Fitness. Int J Exerc Sci. 2017;10(7):1009-17. 